\title{
FLOW OF A NEWTONIAN FLUID IN A SYMMETRICALLY HEATED CHANNEL: EFFECT OF VISCOSITY AND VISCOUS DISSIPATION
}

\author{
K. S. ADEGBIE AND F. I. ALAO \\ Received 15 December 2005; Accepted 21 February 2006
}

This paper discusses the effect of viscosity and viscous dissipation (due to a high velocity gradient) on the steady flow of a viscous liquid in a symmetrically heated channel. The coupled nonlinear differential equations arising in the planar Poiseuille flow are not amendable to analytical solutions. Therefore, numerical solutions based on finitedifference scheme are presented. The effects of various flow controlling parameters such as temperature difference $\alpha$, dimensionless pressure gradient, and the dimensionless viscous heating parameter $\delta$ on the dimensionless velocity and temperature are analyzed. The analysis reveals that when viscous heating parameter $\delta=0$, we obtained zero solution for the dimensionless temperature.

Copyright $\odot 2006$ K. S. Adegbie and F. I. Alao. This is an open access article distributed under the Creative Commons Attribution License, which permits unrestricted use, distribution, and reproduction in any medium, provided the original work is properly cited.

\section{Introduction}

The study of flow of viscous fluid with temperature-dependent properties is of great importance in lubrication and tribology, food processing, instrumentation, and viscometry. However, viscous heating is always a possible, and frequently significant, source of error in viscometric measurement at high shear rates in instrumentation and viscometry. Bird et al. [2], Turian and Bird [8], and Turian [7] have presented a methodology for obtaining approximate analytical solutions to the problem of combine flow and heat transfer in planar Couette flow when both the viscosity and thermal conductivity are polynomial functions of temperature. Papathanasiou [5] developed second-order series solution for flow in circular Couette with walls maintained at constant temperature, for material whose viscosity and thermal conductivity can be expressed as polynomial functions of temperature with arbitrary coefficients. Pinarbasi and Imal [6] investigated the pressuregradient-flow relationship for steady-state nonisothermal pressure-driven flow of a nonNewtonian fluid in a channel and the effect of viscous heating is taken into account. The fluid is modeled as Carreau model, which reflects the characteristics of most polymers

Hindawi Publishing Corporation

Mathematical Problems in Engineering

Volume 2006, Article ID 29314, Pages 1-7

DOI 10.1155/MPE/2006/29314 
2 Flow of a Newtonian fluid in a symmetrically heated channel

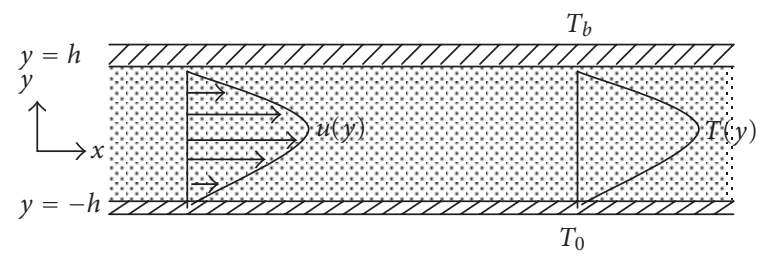

Figure 1.1. Schematic description of physical model.

adequately, with an exponential temperature dependence of viscosity. Momentum and energy balance equations, which govern the flow, are coupled and the nonlinear boundary value problems are solved numerically using a pseudospectral method based on the Chebyshev polynomials. The effects of various flows controlling parameters are analyzed. Davis et al. [3] studied steady parallel flows of Newtonian liquids that have temperaturedependent viscosities and substantial viscous heat generation. They presented shear stress versus shear rate characteristics and found that activation energy parameter affects the results considerably. They observed that shear-stress rate graphs are either monotonic or there exists large jump in shear rate and heat transfer at the walls. Adler [1] investigated the thermal stability of a reactive viscous flow. He considered the steady developed flow between symmetrically heated parallel walls and used a power series in a defined viscous heating parameter to obtain an expression for critical Frank-Kamenestkii parameter in series form. Yürüsoy and Pakdemirli [9] considered the flow of a thirdgrade fluid in a pipe with heat transfer. Constant viscosity, Reynold's model viscosity, and Vogel's model viscosity cases are treated separately. Approximate analytical solutions are presented for each case using perturbations. The criteria for which the solutions are valid are determined for the dimensionless parameters involved. The analytical solutions are contrasted to the finite-difference solutions given by Massoudi and Christiein [4]. The present contribution studies steady-state flow of Newtonian viscous liquid with exponential temperature-dependent viscosity and substantial viscous heat generation in a symmetrically heated channel. The coupled nonlinear momentum and energy equations arising in planar Poiseuille flow are solved numerically using finite-difference scheme techniques. The effects of flow controlling parameters such as viscous heating parameter, temperature difference, and pressure-gradient parameter on the velocity and temperature profiles are analyzed.

\section{Mathematical formulation}

We consider steady flow of Newtonian liquids characterized by temperature-dependent viscosity and viscous dissipation due to a high velocity gradient of the flow in a channel. The main physical assumptions of the suggested problem are the following. The flow is incompressible and fully developed. The body forces are negligible. We presuppose that there is no-slip at the boundary, and pressure changes influence is taken into account. The dependence of thermal conductivity on temperature is neglected. The viscosity depends 
on temperature in an exponential manner, Reynold's model viscosity,

$$
\mu(T)=\exp (-T)
$$

Under these assumptions, the system of governing dimensionless momentum balance and energy balance equations take the following form:

$$
\begin{gathered}
\frac{d}{d r}\left[\exp (-\alpha \theta) \frac{d u}{d r}\right]=K \\
\frac{d^{2} \theta}{d r^{2}}+B_{r m} \exp (-\alpha \theta)\left(\frac{d u}{d r}\right)^{2}=0,
\end{gathered}
$$

subject to the following boundary conditions:

$$
\begin{array}{ll}
u(-1)=0, & u(1)=1, \\
\theta(-1)=0, & \theta(1)=0 .
\end{array}
$$

The above coupled nonlinear flow governing equations were made dimensionless using

$$
\theta=\frac{T-T_{0}}{T_{b}-T_{0}}, \quad u=\frac{\bar{u}}{U_{0}}, \quad r=\frac{y}{h}, \quad \mu=\frac{\bar{\mu}}{\mu_{0}}, \quad P=\frac{\bar{P} h}{\mu_{0} U_{0}} .
$$

The dimensionless parameters involved in (2.2)-(2.3) are

$$
K=h \exp \left(T_{0}\right) \frac{d P}{d x}, \quad \alpha=T_{S}-T_{0}, \quad B_{r m}=\left(\frac{\mu_{0} U_{0}^{2}}{\alpha \lambda}\right) \exp \left(-T_{0}\right),
$$

where $B_{r m}$ is the modified Brinkman number, $B_{r}=\left(\mu_{0} U_{0}^{2} / \alpha \lambda\right)$ is Brinkman number, which is a measure of heat generated by viscous heating as compared to the heat conducted from the impressed temperature difference $\alpha$ between the outer and inner surfaces, respectively, through the viscous liquid, $K$ is the pressure-gradient parameter, $\theta$ is the dimensionless temperature, $u$ is the velocity parallel to the planes, $U_{0}$ is the constant velocity at moving surface, $\mu_{0}$ is the viscosity at $T_{0}$, and $P$ is the pressure.

\section{Method of solution}

Here, we consider the solutions of flow of viscous liquid with a high velocity gradient in presence of pressure gradient when its viscosity is an exponential function of temperature. For Poiseuille flow, the pressure gradient is constant, $K \neq 0$. Thus, (2.2) and (2.3) are solved with the symmetric boundary conditions

$$
\begin{array}{ll}
u(1)=0, & \frac{d u(0)}{d r}=0, \\
\theta(1)=0, & \frac{d \theta(0)}{d r}=0 .
\end{array}
$$

Integration of (2.2) using (3.1) yields

$$
\frac{d u}{d r}=K r \exp (\alpha \theta)
$$


4 Flow of a Newtonian fluid in a symmetrically heated channel

Table 4.1. Variation of maximum velocity with pressure gradient for various values of temperature difference $\alpha$ when value of $\delta=-2$.

\begin{tabular}{l|rrrrr}
\hline \multirow{2}{*}{$\alpha$} & \multicolumn{5}{|c}{$u_{\max }$} \\
\cline { 2 - 6 } & $K=-1$ & $K=-2$ & $K=-3$ & $K=-4$ & $K=-5$ \\
\hline 1 & 0.6375 & 1.2750 & 1.1925 & 2.5500 & 3.1875 \\
2 & 0.7702 & 1.5405 & 2.3107 & 3.0809 & 3.8512 \\
3 & 0.9946 & 1.9892 & 2.9838 & 3.9784 & 4.9730 \\
5 & 2.7516 & 5.5031 & 8.2547 & 11.0063 & 13.7578 \\
6 & 13.8306 & 27.6611 & 41.4917 & 55.3222 & 69.1528 \\
\hline
\end{tabular}

Then, substitution of (3.2) into (2.3) gives

$$
\frac{d^{2} \theta}{d r^{2}}+\delta r^{2} \exp (\alpha \theta)=0
$$

where $\delta=K^{2} B_{r m}$. The dimensionless parameter $\delta$ is a measure of

(i) the extent to which viscous heating is important relative to heat flow resulting from impressed temperature difference $\alpha$;

(ii) the effect of pressure gradient on the system;

(iii) the effect of inner temperature on the system;

(iv) what the dimension of the system involves.

Now, the nonlinear boundary value problems (3.2) and (3.3) with conditions in (3.1) above are solved numerically using finite-difference scheme techniques.

\section{Numerical analysis and discussion}

In this section, numerical solutions of plane Poiseuille flow are presented. Table 4.1 shows the variation of maximum velocity with the value of applied pressure gradient for various values of temperature difference $\alpha$ when the values of dimensionless viscous heating parameter $\delta=-2$. It is obvious from Table 4.1 that the increase in maximum velocity with decreasing pressure gradient is monotonic for all values of $\alpha$. However, there is a sudden jump from $u_{\max }=0.9946$ to $u_{\max }=2.7516$ when $\alpha$ is increased from $\alpha=3$ to 5 for $K=-1$. This jump in the maximum velocity is more pronounced as $K$ decreases. When $K=-5$, a sharp sudden jump from $u_{\max }=13.7578$ to $u_{\max }=69.1528$ occurs when $\alpha$ is increased from $\alpha=5$ to 6 . Conclusively, the liquids whose viscosity is not very temperature-sensitive do not experience large jumps in the maximum velocity as applied pressure gradient decreases.

We show in Table 4.2 typical variations of maximum temperature $\theta_{\max }$ and maximum velocity $u_{\max }$ with various values of $\delta$ when $K=-1$. It is seen from Table 4.2 that for $\alpha=1$, the characteristic curves are monotonically increasing, and there are no jumps in both $\theta_{\max }$ and $u_{\max }$. However, when $\alpha=3$ and $\alpha=4$, the temperature and velocity increase smoothly as $\delta$ decreases until a critical point is reached, there is a large jump in $\theta_{\max }$ and $u_{\max }$, respectively. 
Table 4.2. Variation of maximum temperature and maximum velocity with dimensionless parameter $\delta$ for various values of $\alpha$ when $K=-1$.

\begin{tabular}{l|rrrr|rrrr}
\hline \multirow{2}{*}{$\delta$} & \multicolumn{4}{|c|}{$\theta_{\max }$} & \multicolumn{4}{c}{$u_{\max }$} \\
\cline { 2 - 9 } & $\alpha=1$ & $\alpha=2$ & $\alpha=3$ & $\alpha=4$ & $\alpha=1$ & $\alpha=2$ & $\alpha=3$ & $\alpha=4$ \\
\hline-0.1 & 0.0303 & 0.0304 & 0.0305 & 0.0306 & 0.5537 & 0.5574 & 0.5613 & 0.5650 \\
-0.5 & 0.1535 & 0.1559 & 0.1585 & 0.1612 & 0.5690 & 0.5897 & 0.6125 & 0.6375 \\
-1 & 0.3118 & 0.3225 & 0.3341 & 0.3477 & 0.5897 & 0.6375 & 0.6963 & 0.7702 \\
-2 & 0.6446 & 0.6953 & 0.7641 & 0.8661 & 0.6375 & 0.7702 & 0.9946 & 1.4479 \\
-3 & 1.0024 & 1.1461 & 1.4115 & 2.4540 & 0.6963 & 0.9946 & 1.8967 & 13.8306 \\
-5 & 1.8170 & 2.6193 & 4.0726 & 19.8964 & 0.8660 & 2.7516 & 12.3479 & 88.4611 \\
\hline
\end{tabular}

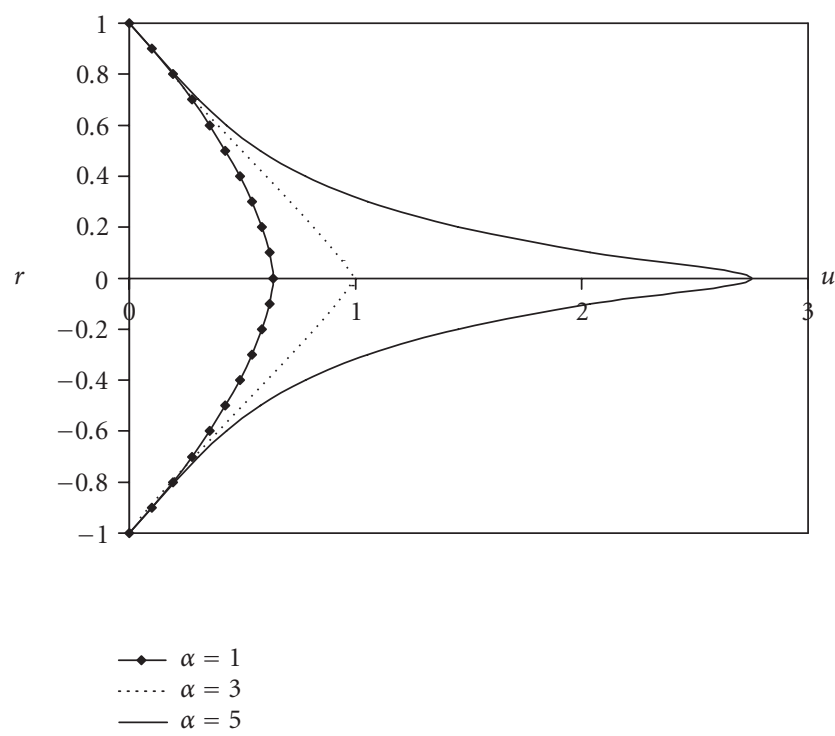

Figure 4.1. Variation of velocity profile with various temperature difference parameters $\alpha$ when $K=$ -1 and $\delta=-2$.

Figure 4.1 shows the dimensionless velocity profile for various temperature difference parameters $\alpha$ for $\delta=-2$ and $K=-1$. It is apparent that the velocity profiles are symmetric with respect to the midpoint, and the two inflection points that are symmetrically located are seen in Figure 4.1. For $\alpha=1$ and $\alpha=3$ shown in Figure 4.1, there is a small jump and therefore the values of the velocity are small. However, when $\alpha=5$, velocity values are high due to the jump that occurred in the maximum velocity. Therefore, an increase in $\alpha$ signifies the sensitivity of the liquid to temperature. Since the viscosity of the liquids decreases with increasing $\alpha$, the dimensionless velocity profile takes higher values as $\alpha$ increases. In Figures 4.2 and 4.3, the results in Table 4.2 are ascertained. As expected, the dimensionless maximum temperature and velocity increase monotonically as $\delta$ decreases. 
6 Flow of a Newtonian fluid in a symmetrically heated channel

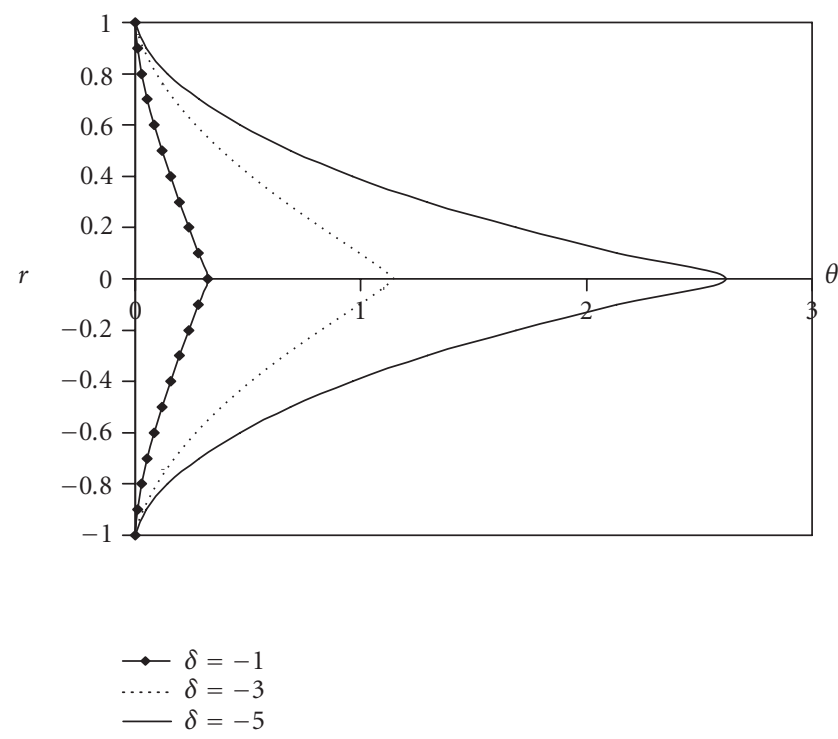

Figure 4.2. Variation of temperature profile with the values of temperature difference parameter $\alpha=2$ for various values of $\delta$ when $K=-1$.

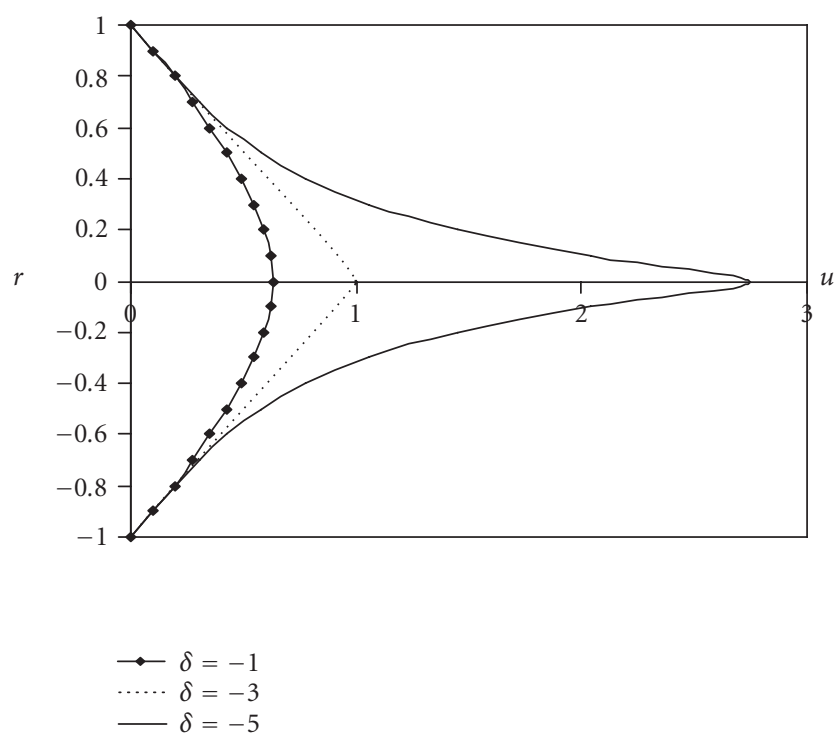

Figure 4.3. Variation of dimensionless velocity profile with the value of temperature difference parameter $\alpha=2$ for various values of $\delta$ when $K=-1$. 


\section{Conclusion}

Steady flow of Newtonian fluid with exponential temperature-dependent viscosity and viscous dissipation in a symmetrically heated channel is investigated. The numerical solutions of the coupled nonlinear equations arising from planar Poiseuille flow are also presented. The effects of various flow controlling parameters such as temperature difference $\alpha$, dimensionless pressure gradient, and the dimensionless parameter $\delta$ on the dimensionless velocity and temperature are analyzed. The graphical representations of these effects feature promptly. It is found that the behavior of the solutions is replica of some of the problems in lubrication, viscometry, and engineering flows such as pumping of oil in pipes, flow of liquids in open channels, and extrusion of plastics through dies.

\section{References}

[1] J. Adler, Thermal explosion theory for reactive flow between parallel heated walls, Combustion and Flame 24 (1975), 151-158.

[2] R. B. Bird, W. E. Stewart, and E. N. Lightfoot, Transport Phenomena, John Wiley \& Sons, New York, 1960.

[3] S. H. Davis, G. A. Kriegsmann, R. L. Laurence, and S. Rosenblat, Multiple solutions and hysteresis in steady parallel viscous flows, Physics of Fluids 26 (1983), no. 5, 1177-1182.

[4] M. Massoudi and I. Christie, Effects of variable viscosity and viscous dissipation on the flow of a third grade fluid in a pipe, International Journal of Non-Linear Mechanics 30 (1995), no. 5, 687-699.

[5] T. D. Papathanasiou, Circular Couette flow of temperature-dependent materials: asymptotic solutions in the presence of viscous heating, Chemical Engineering Science 52 (1997), no. 12, 20032006.

[6] A. Pinarbasi and M. Imal, Nonisothermal channel flow of a non-Newtonian fluid with viscous heating, International Communications in Heat and Mass Transfer 29 (2002), no. 8, 1099-1107.

[7] R. M. Turian, Viscous heating in the cone-and-plate viscometer-III. Non-Newtonian fluids with temperature-dependent viscosity and thermal conductivity, Chemical Engineering Science 20 (1965), no. 8, 771-781.

[8] R. M. Turian and R. B. Bird, Viscous heating in the cone-and-plate viscometer-II. Newtonian fluids with temperature-dependent viscosity and thermal conductivity, Chemical Engineering Science 18 (1963), no. 11, 689-696.

[9] M. Yürüsoy and M. Pakdemirli, Approximate analytical solutions for the flow of a third-grade fluid in a pipe, International Journal of Non-Linear Mechanics 37 (2002), no. 2, 187-195.

K. S. Adegbie: Department of Mathematical Sciences, School of Sciences, Federal University of Technology, P.M.B. 704, Akure, Ondo State, Nigeria E-mail address: kolasade33@yahoo.com

F. I. Alao: Department of Mathematical Sciences, School of Sciences, Federal University of Technology, P.M.B. 704, Akure, Ondo State, Nigeria E-mail address: dupsan65@yahoo.com 


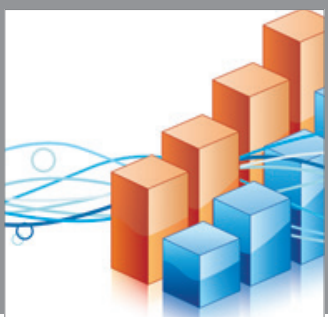

Advances in

Operations Research

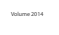

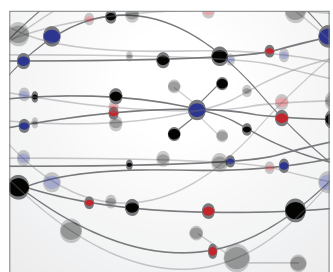

\section{The Scientific} World Journal
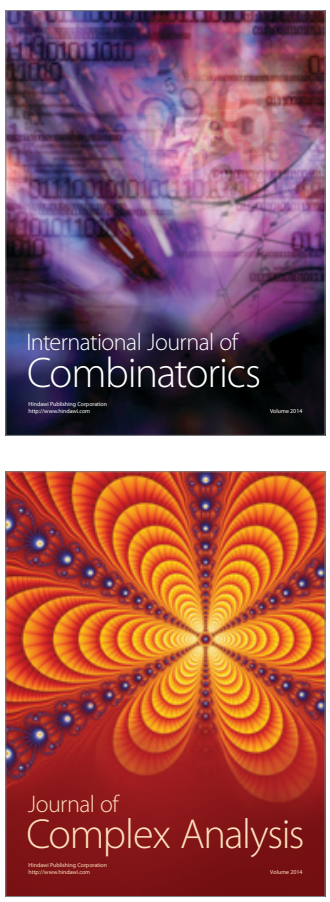

International Journal of

Mathematics and

Mathematical

Sciences
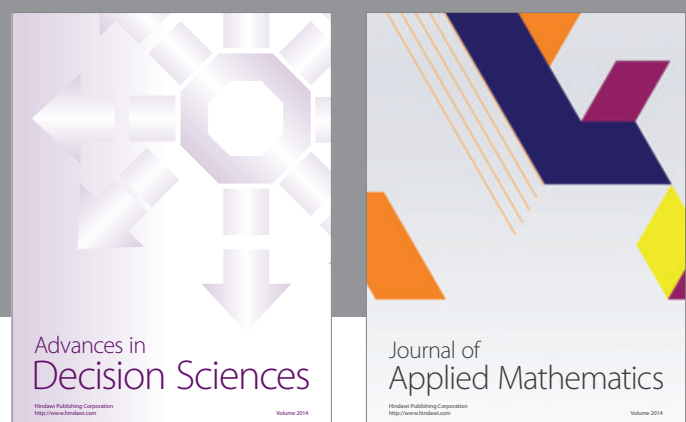

Journal of

Applied Mathematics
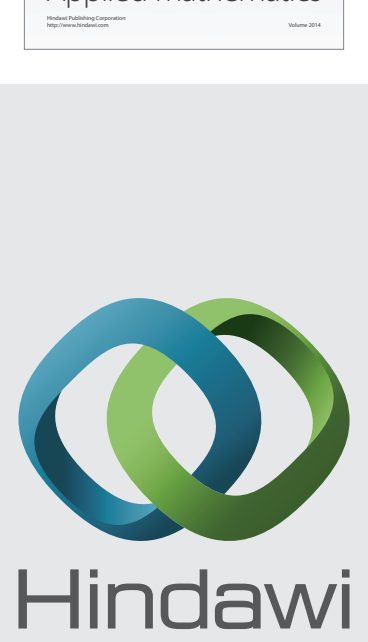

Submit your manuscripts at http://www.hindawi.com
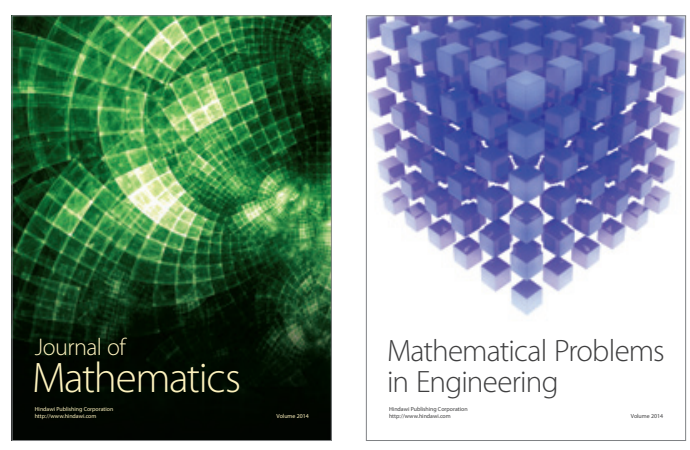

Mathematical Problems in Engineering
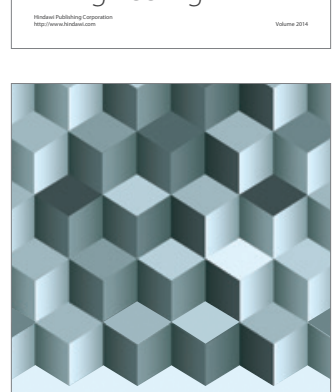

Journal of

Function Spaces
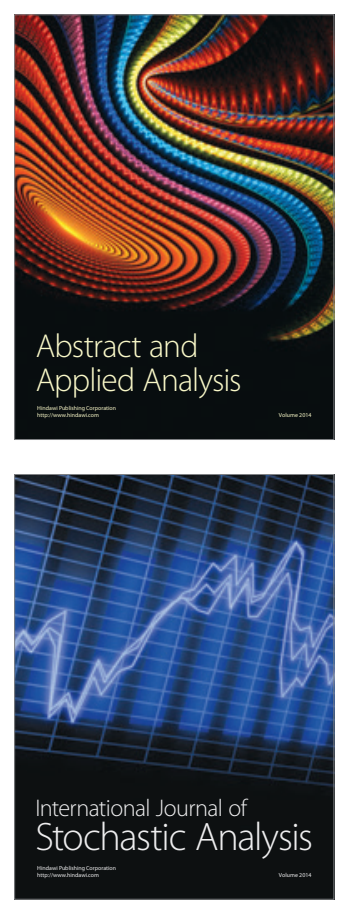

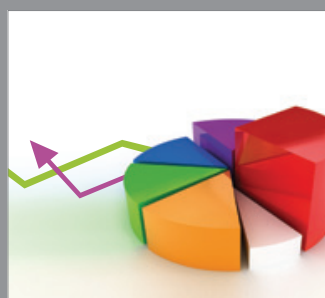

ournal of

Probability and Statistics

Promensencen
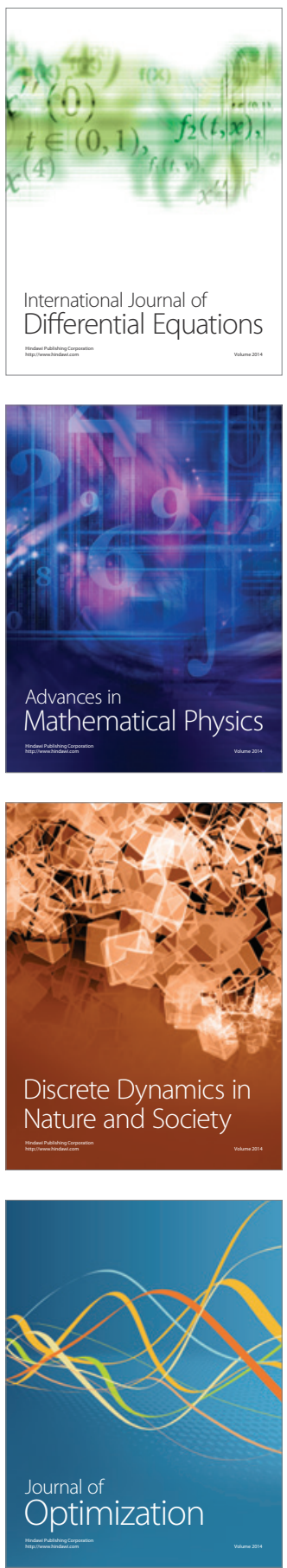